University of Warwick institutional repository: http://go.warwick.ac.uk/wrap This paper is made available online in accordance with publisher policies. Please scroll down to view the document itself. Please refer to the repository record for this item and our policy information available from the repository home page for further information.

To see the final version of this paper please visit the publisher's website. Access to the published version may require a subscription.

Author(s): M.A. ALLEN, S. PHIBANCHON and G. ROWLANDS Article Title: Weakly nonlinear waves in magnetized plasma with a slightly non-Maxwellian electron distribution. Part 1. Stability of solitary waves

Year of publication: 2007

Link to published version: http://dx.doi.org/

10.1017/S0022377806004508

Publisher statement: None 


\title{
Weakly nonlinear waves in magnetized plasma with a slightly non-Maxwellian electron distribution. Part 1. Stability of solitary waves
}

\author{
M. A. A LLEN ${ }^{1}$, S. PHIBA NCHON $\mathrm{N}^{2}$ and G. ROWLAN D S \\ ${ }^{1}$ Physics Department, Mahidol University, Rama 6 Road, Bangkok 10400, Thailand \\ ${ }^{2}$ Faculty of Sciences and Liberal Arts, Burapha University, 57 Moo 1, Thamai, \\ Chanthaburi 22170, Thailand \\ ${ }^{3}$ Department of Physics, University of Warwick, Coventry CV4 7AL, UK \\ (frmaa@mahidol.ac.th; sarunpb@yahoo.co.uk; G. Rowlands@warwick.ac.uk)
}

(Received 21 February 2006)

\begin{abstract}
Weakly nonlinear waves in strongly magnetized plasma with slightly non-isothermal electrons are governed by a modified Zakharov-Kuznetsov (ZK) equation, containing both quadratic and half-order nonlinear terms, which we refer to as the Schamel-Korteweg-de Vries-Zakharov-Kuznetsov (SKdVZK) equation. We present a method to obtain an approximation for the growth rate, $\gamma$, of sinusoidal perpendicular perturbations of wavenumber, $k$, to SKdVZK solitary waves over the entire range of instability. Unlike for (modified) ZK equations with one nonlinear term, in this method there is no analytical expression for $k_{\mathrm{c}}$, the cut-off wavenumber (at which the growth rate is zero) or its corresponding eigenfunction. We therefore obtain approximate expressions for these using an expansion parameter, $a$, related to the ratio of the nonlinear terms. The expressions are then used to find $\gamma$ for $k$ near $k_{\mathrm{e}}$ as a function of $a$. The approximant derived from combining these analytical results with the ones for small $k$ agrees very well with the values of $\gamma$ obtained numerically. It is found that both $k_{\mathrm{c}}$ and the maximum growth rate decrease as the electron distribution becomes progressively less peaked than the Maxwellian. We also present new algebraic and rarefactive solitary wave solutions to the equation.
\end{abstract}

\section{Introduction}

Small amplitude nonlinear waves in a cold-ion plasma in which there is a uniform magnetic field whose strength is sufficient for the Alfvén velocity to be comparable to the speed of light are governed by the Zakharov-Kuznetsov (ZK) equation,

$$
u_{t}+u u_{x}+\nabla^{2} u_{x}=0
$$

in which the subscripts denote derivatives, $u$ is proportional to the electrostatic potential which itself is proportional to the deviation from the mean ion density, and $x$ is a normalized coordinate in a frame moving at the speed of long-wavelength linear acoustic waves in the direction of the magnetic field (Zakharov and Kuznetsov 1974). For a system with no $y$ or $z$-dependence, (1.1) reduces to the well-known Korteweg-de Vries $(\mathrm{KdV})$ equation which also describes such waves in the absence of a magnetic field (Washimi and Tanuiti 1966). 
In the derivations of the $\mathrm{KdV}$ and $\mathrm{ZK}$ equations for cold-ion plasmas, it is assumed that the electrons have a Maxwellian distribution. In the case where the electrons make numerous collisions with each other or with the boundaries of the vessel containing the plasma, this assumption is reasonable. However, if the effect of the collisions is small, the electrons will no longer be isothermal. An improved description of the electron behaviour can be obtained by assuming that the electron distribution, which is described by a Vlasov equation, instantaneously relaxes to a BGK mode (Bernstein et al. 1957). Using this result, if both the trapped and free particles have Maxwellian distributions but with different temperatures, it can be shown that one-dimensional weakly nonlinear ion-acoustic waves are governed by the Schamel equation

$$
u_{t}+|u|^{1 / 2} u_{x}+u_{x x x}=0
$$

(Schamel 1973). The equation also applies to electron and ion holes resulting from particle trapping in which case the underlying acoustic waves are, respectively, the so-called slow electron and ion-acoustic modes rather than the ordinary ion-acoustic waves (Schamel 1986). For the magnetized plasma case, the equation

$$
u_{t}+|u|^{1 / 2} u_{x}+\nabla^{2} u_{x}=0
$$

has been obtained (Shukla and Bharuthram 1986; Ghosh and Das 1998; Munro and Parkes 1999). To distinguish it from other modified ZK equations, we will refer to (1.2) as the Schamel-Zakharov-Kuznetsov (SZK) equation. As explained in Schamel (1973), in the above and subsequent equations involving the square root of $|u|$, it should be noted that $u$ is only permitted to be slightly negative. All the analytical solutions are thus for $u \geqslant 0$ with the result that the modulus sign is generally omitted in the literature and will be dropped from Sec. 2 onwards in this article. However, in numerical calculations of, for example, solitary wave collisions, small oscillations resulting in negative $u$ occur which therefore necessitate its inclusion.

It has been pointed out that if the two electron distributions differ only slightly in temperature, then one obtains an equation with both quadratic and half-order nonlinearities, namely, the Schamel-KdV (SKdV) equation (Schamel 1973),

$$
u_{t}+\left(u+b|u|^{1 / 2}\right) u_{x}+u_{x x x}=0
$$

in which

$$
b=\frac{1}{\sqrt{\pi}}\left(1-\frac{T_{\mathrm{ef}}}{T_{\mathrm{et}}}\right)
$$

where $T_{\text {ef }}$ and $T_{\text {et }}$ are the effective temperatures of the free and trapped electrons, respectively. In addition to being used to describe larger amplitude solitary waves (Schamel 1972), the equation has since been shown to apply to dust-acoustic solitary waves with trapped electrons (El-Labany and El-Taibany 2004) and pulse-shaped perturbations in particle accelerator beams (Schamel and Fedele 2000).

We address the case of weakly nonlinear waves in a cold-ion, strongly magnetized plasma with two populations of Maxwell distributed electrons at slightly different temperatures, and use a modified $\mathrm{ZK}$ equation that takes the form

$$
u_{t}+\left(u+b|u|^{1 / 2}\right) u_{x}+\nabla^{2} u_{x}=0
$$

As in the case of the SKdV equation, in deriving this equation, it is assumed that $b=O\left(|u|^{1 / 2}\right)$ and that the normalized deviation from mean ion density, $u$, is small 
(Shukla and Bharuthram 1986). Since (1.3) reduces to the SKdV equation in one dimension, we refer to it as the SKdVZK equation. Its solitary wave solutions are discussed in Sec. 2. The remainder of the paper is concerned with studying the stability of a solitary wave solution with respect to perturbations applied at an angle to the direction of propagation. As far as we know, this is the first time such a calculation has been performed for an equation with two nonlinear terms, although a number of modified ZK equations with multiple nonlinearities have appeared in the literature (Das and Sen 1991; Das et al. 2000; Verheest et al. 2002).

Linear stability analysis for both the ZK and SZK equations reveals that there is a cut-off perturbation wavenumber, $k_{\mathrm{c}}$, above which the solitary wave solution is stable (Laedke and Spatschek 1982; Munro and Parkes 2000), and the growth rate of instabilities for perturbation wavenumber $k$ less than $k_{\mathrm{c}}$ has been determined (Allen and Rowlands 1993; Munro and Parkes 2000). In Sec. 3 we find the growth rate of small- $k$ perturbations to SKdVZK solitary waves using a small- $k$ expansion method. To obtain the growth rate for larger values of $k$, we first need to find $k_{\mathrm{c}}$. When the growth rate is zero, both the linearized ZK and SZK equations can be written in standard form with the result that it is straightforward to determine $k_{\mathrm{c}}$. The linearized SKdVZK equation does not correspond to any solved eigenvalue problem, and so we must find an approximate form for $k_{\mathrm{c}}$ as a function of a parameter, $a$, which is related to the ratio of the nonlinearities. This is done by expanding about the two known solutions to the eigenvalue problem (which correspond to the ZK and SZK limiting cases) and is dealt with in Sec. 4. Having obtained an approximate solution to the eigenvalue problem in the neighbourhood of the ZK and SZK limits in the form of expansions in which the small parameter is related to $a$, in Sec. 5 we find the growth rate for values of $k$ close to $k_{\mathrm{c}}$ by expanding in $k_{\mathrm{c}}-k$ about this approximate solution. The growth rate results for the various values of $k$ and $a$ are combined using a Padé approximant and compared with numerically derived values in Sec. 6 . In the final section we summarize our results and discuss the applicability of the method.

\section{Solitary wave solutions}

To look for plane solitary wave solutions travelling at speed $V$ in the $x$-direction, we apply the transformation $x^{\prime}=x-V t$ to (1.3) and then drop the $t, y$ and $z$ dependence. Integrating, multiplying by $2 u_{x}$, integrating again, and then dropping the primes gives

$$
u_{x}^{2}=C_{0}+C u+V u^{2}-\frac{8}{15} b u^{5 / 2}-\frac{1}{3} u^{3}
$$

where $C_{0}$ and $C$ are arbitrary constants. Sketches of $(2.1)$ giving examples from families of curves that will lead to solitary wave solutions and necessary conditions on $C_{0}, C, b$ and $V$ for their existence are given in Fig. 1 .

It can be seen that all the solitary waves are pulse-shaped. For $b>0$, there are two families of such solutions. Figures $1(a)$ and (b) correspond, respectively, to solitary waves that vanish or remain finite as $|x| \rightarrow \infty$. In the former case, Schamel (1972) has given an explicit expression for the solution which we prefer to write in the form

$$
u(x)=\frac{12 \eta^{2}\left(1-a^{2}\right)}{(a+\cosh \eta x)^{2}}
$$



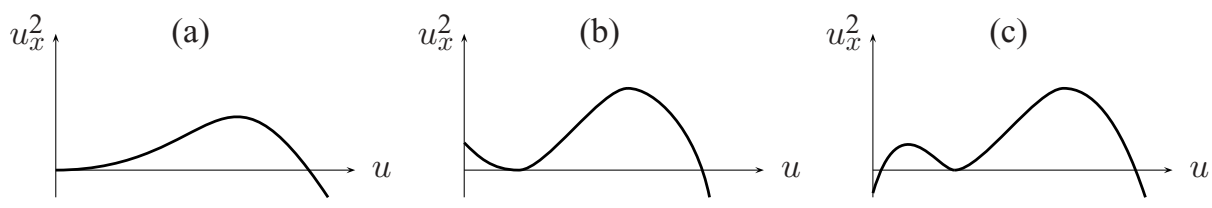

Figure 1. $\left(u, u_{x}^{2}\right)$-sketches of members of SKdVZK solitary wave solution families: (a) $C_{0}=C=0, V \geqslant 0$ (when $V=0, b<0$ ); (b) $C_{0}>0, C<0, V>0$; (c) $V<0, b<0$.

where $\eta=\sqrt{V} / 2$ and

$$
a=\frac{2 b}{\sqrt{75 \eta^{2}+4 b^{2}}} .
$$

It can be seen that when $b=0,(2.2)$ reduces to the familiar $12 \eta^{2} \operatorname{sech}^{2} \eta x$ solution of the $\mathrm{KdV}$ equation. In the limit of large $b, a \rightarrow 1$ and the Schamel equation solitary wave solution

$$
u(x) \rightarrow \frac{225 \eta^{4}}{4 b^{2}} \operatorname{sech}^{4} \frac{\eta x}{2}
$$

is recovered. It has been shown that the Schamel equation is not integrable (Ramani and Grammaticos 1991), from which it can be inferred that this is also the case for the SKdV equation. Nevertheless, numerical experiments we have performed show that a hump-shaped initial condition evolves into a train of solitary waves of the form (2.2). Such solitary waves can also pass through one another with phase changes in a similar way to $\mathrm{KdV}$ solitons.

When $b<0$, solitary waves corresponding to all three graphs in Fig. 1 are possible. The most interesting case is when $C_{0}=C=V=0$. Solving (2.1) then gives an algebraically decaying non-singular solitary wave solution which we believe has not been reported previously. It takes the form

$$
u(x)=\frac{900 / b^{2}}{\left(75 / 4 b^{2}+x^{2}\right)^{2}} .
$$

This solution can also be obtained from (2.2) in the limit $\eta \rightarrow 0$ for $b<0$. Non-singular algebraic soliton solutions also occur for the modified $\mathrm{KdV}$ equation (Ono 1976), and the extended KdV equation which has both quadratic and cubic nonlinearities (Pelinovsky and Grimshaw 1997). The solution (2.3) is similar to the latter case in that it vanishes as $|x| \rightarrow \infty$ and it is stationary (in the frame moving with long-wavelength linear waves). Our numerical experiments suggest that these algebraic solitary waves are stable in the sense that they survive collisions with conventional (exponentially decaying) solitary waves.

After employing the tanh method to solve (1.3) written in terms of $u^{1 / 2}$, Das et al. (2000) give two solitary wave solutions which in the $y$ and $z$-independent forms reduce to $u^{1 / 2}=-\sqrt{6} \pm \sqrt{12} \operatorname{sech}(x+8 t)$ if $b=\sqrt{75 / 2}$. These do not appear in the phase plane analysis and are erroneous since in (1.3) only a positive square root can be taken. However, if $b=-\sqrt{75 / 2}$, their solution becomes $u^{1 / 2}=\sqrt{6}+$ $\sqrt{12} \operatorname{sech}(x+8 t)$ which is permissible and corresponds to a member of a family of solutions of $(2.1)$ with $b<0$ given by

$$
u^{1 / 2}(x)=-\frac{2 b}{5}\left[1+\sqrt{2} \operatorname{sech}\left(\sqrt{\frac{2}{75}} b x\right)\right]
$$


for which $C_{0}=0, C=12\left(2 b^{2} / 75\right)^{3}, V=-16 b^{2} / 75$. For these values of the parameters there is another family of solitary wave solutions which has not been mentioned elsewhere. They take the form

$$
u^{1 / 2}(x)=-\frac{2 b}{5}\left[1-\sqrt{2} \operatorname{sech}\left(\sqrt{\frac{2}{75}}|b x|+\cosh ^{-1} \sqrt{2}\right)\right]
$$

and are therefore rarefactive. In Fig. 1(c) members of this family correspond to the curve joining the origin (since $C_{0}=0$ ) to the double root at $u=4 b^{2} / 25$.

As with the ZK and SZK equations, the SKdVZK equation also admits obliquely propagating solitary wave solutions. These have the same form as the various solutions given above with $x$ replaced by $l x+m y+n z$ where $l, m, n$ are the direction cosines. Their analysis will be deferred until a future article.

From the original derivation of the Schamel equation it is seen that as $b$ becomes more negative, the electron distribution becomes more peaked. Distributions that are more peaked than the Maxwellian are contrary to numerical and experimental evidence (Schamel 1973). Thus in the remainder of this paper, we focus on the stability of the most physically relevant of the solitary wave solutions, namely, (2.2) for $0<a<1$. However, in addition, the stability of the algebraic solitary wave (which corresponds to the limit $a \rightarrow-1, \eta \rightarrow 0$ ) is briefly discussed in Appendix A.

\section{Growth rate for small- $k$ instabilities}

After transforming (1.3) to a frame moving along the $x$-axis at speed $4 \eta^{2}$, making the change of variables,

$$
x^{\prime}=\eta x, \quad y^{\prime}=\eta y, \quad z^{\prime}=\eta z, \quad t^{\prime}=\eta^{3} t, \quad u^{\prime}=u / \eta^{2}, \quad b^{\prime}=b / \eta,
$$

and then dropping the primes, we obtain

$$
u_{t}+\left(u+b u^{1 / 2}-4\right) u_{x}+\nabla^{2} u_{x}=0
$$

To investigate the stability of the plane solitary wave,

$$
u_{0}(x)=\frac{12\left(1-a^{2}\right)}{(a+\cosh x)^{2}}
$$

with respect to a perturbation of wavenumber $k(\cos \theta, \sin \theta \cos \lambda, \sin \theta \sin \lambda)$ we substitute the ansatz

$$
u=u_{0}(x)+\varepsilon \phi(x) e^{i k(x \cos \theta+y \sin \theta \cos \lambda+z \sin \theta \sin \lambda)} e^{\gamma t}
$$

where $\varepsilon \ll 1$ into (3.1). Then to first order in $\varepsilon$ we obtain

$$
\frac{d}{d x} L \phi=-\gamma \phi-i k \cos \theta Q \phi-3 i k \cos \theta \phi_{x x}+k^{2}\left(1+2 \cos ^{2} \theta\right) \phi_{x}+i k^{3} \cos \theta \phi
$$

where

$$
L \equiv \frac{d^{2}}{d x^{2}}+Q, \quad Q \equiv \frac{12+3 a^{2}+15 a \cosh x}{(a+\cosh x)^{2}}-4 .
$$

We expand $\phi$ and the growth rate $\gamma$ in $k$ as follows:

$$
\begin{gathered}
\phi=\phi_{0}+k \phi_{1}+k^{2} \phi_{2}+\cdots, \\
\gamma=\gamma_{1} k+\gamma_{2} k^{2}+\cdots
\end{gathered}
$$


To zeroth order in $k,(3.4)$ gives

$$
\frac{d}{d x} L \phi_{0}=0
$$

Since the solution to (3.1) can be translated in the $x$-direction, it is clear from setting $k=0$ in (3.3) that $\phi_{0}$ is proportional to $u_{0 x}$. Hence

$$
\phi_{0}=\frac{\sinh x}{(a+\cosh x)^{3}}
$$

and we are then able to calculate the inverse of $L$ via

$$
L^{-1} R(x)=\phi_{0}(x) \int^{x} \frac{\int^{x^{\prime}} R\left(x^{\prime \prime}\right) \phi_{0}\left(x^{\prime \prime}\right) d x^{\prime \prime}}{\phi_{0}^{2}\left(x^{\prime}\right)} d x^{\prime} \bmod \phi_{0}(x)
$$

as in Allen and Rowlands (1993) (which will henceforth be referred to as AR93). To first order in $k$, after using $\phi_{0 x x}+Q \phi_{0}=0$, we have

$$
\frac{d}{d x} L \phi_{1}=-\gamma_{1} \phi_{0}-2 i \cos \theta \phi_{0 x x}
$$

from which we obtain

$$
\phi_{1}=\frac{\gamma_{1}}{8} \frac{2 a+\left(1+a^{2}\right) \cosh x-x \sinh x}{(a+\cosh x)^{3}}-i \cos \theta x \phi_{0} .
$$

The solution of the second-order equation

$$
\frac{d}{d x} L \phi_{2}=-\gamma_{2} \phi_{0}-\gamma_{1} \phi_{1}+\left(1+2 \cos ^{2} \theta\right) \phi_{0 x}-i \cos \theta Q \phi_{1}-3 i \cos \theta \phi_{1 x x}
$$

is extremely complicated. Moreover, $\phi_{n}$ for $n \geqslant 2$ contain algebraic secularities that need to be regrouped to obtain a consistent expansion at higher order as shown in AR93. In AR93, $\gamma_{1}$ was obtained by eliminating exponential secularities in $\phi_{2}$. However, $\gamma_{1}$ can be obtained without evaluating $\phi_{2}$ by applying a consistency condition instead (Allen 1994; Infeld and Rowlands 2000).

The consistency condition is obtained by multiplying (3.6) by $\int^{x} \phi_{0} d x$ and integrating over all $x$. The left-hand side can be seen to equal zero after integrating by parts and using the self-adjoint property of $L$ and the fact that $L \phi_{0}=0$. The first term on the right-hand side also vanishes as the integrand is odd. The result can then be reduced to

$$
\frac{\gamma_{1}^{2}}{8} \int_{-\infty}^{\infty} \frac{2 a+\left(1+a^{2}\right) \cosh x-x \sinh x}{(a+\cosh x)^{5}} d x=\sin ^{2} \theta \int_{-\infty}^{\infty} \frac{\sinh ^{2} x}{(a+\cosh x)^{6}} d x .
$$

The integrals may be performed using contour integration as detailed in Appendix B and we obtain

$$
\gamma_{1}^{2}=\frac{8}{15}\left\{\frac{\sqrt{1-a^{2}}\left(16+83 a^{2}+6 a^{4}\right)-15 a\left(3+4 a^{2}\right) \cos ^{-1} a}{\left(1-a^{2}\right)^{2}\left(\sqrt{1-a^{2}}\left(2+a^{2}\right)-3 a \cos ^{-1} a\right)}\right\} \sin ^{2} \theta .
$$

The fact that the expression for $\gamma_{1}$ vanishes when $\theta=0$ is in agreement with our tacit assumption, backed by numerical experiments, that the wave is stable to perturbations along the direction of propagation, as is the case for the $\mathrm{KdV}$ and Schamel equations. The maximum growth rate for a given $a$ is for perpendicular perturbations $(\theta=\pi / 2)$. From now on we therefore only consider this case. Putting $a=0$ in (3.7) gives $\gamma_{1}^{2}=64 / 15$, as was first obtained by Laedke and Spatschek 


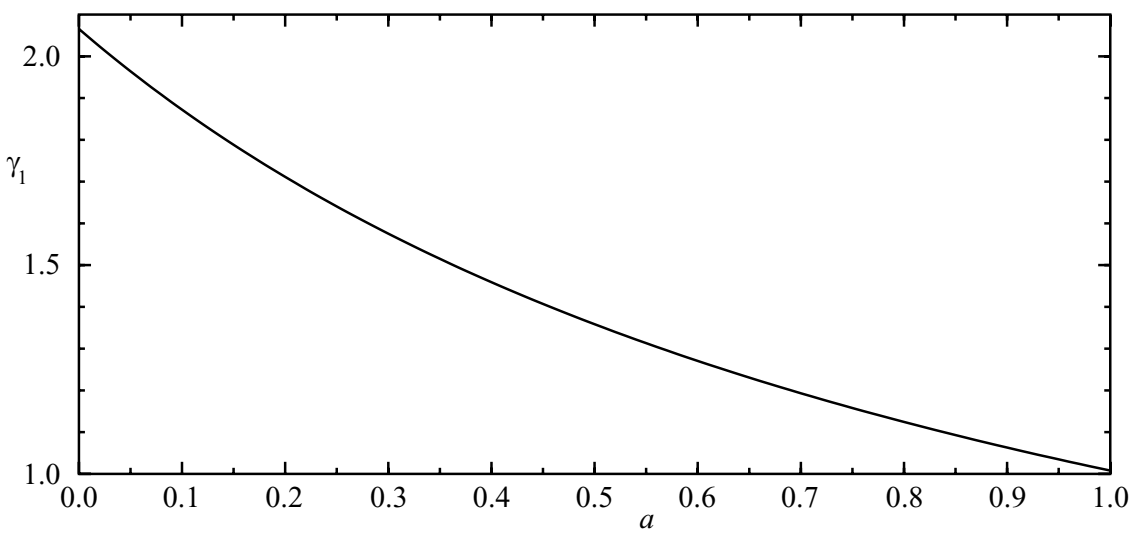

Figure 2. The dependence of $\gamma_{1}$ on $a$.

(1982) for the ZK equation. As shown in Fig. 2, the growth rate decreases monotonically with increasing $a$ up to $a=1$ at which point $\gamma_{1}^{2}=64 / 63$, which after rescaling is in agreement with the result previously found for the SZK equation (Munro and Parkes 2000).

\section{Determination of the cut-off wavenumber}

We expect that for a critical value of $k$, namely the cut-off wavenumber $k_{\mathrm{c}}(a)$, the solution is stable for $k \geqslant k_{\mathrm{c}}(a)$. Since $\gamma=0$ when $k=k_{\mathrm{c}}(a)$, the value of $k_{\mathrm{c}}(a)$ can be determined by solving the eigenvalue problem

$$
\left(L-k_{\mathrm{c}}^{2}(a)\right) \phi_{\mathrm{e}}(a)=0
$$

obtained by integrating (3.4) with $\theta=\pi / 2$. When $a=0$ or $a=1$, the equation can be written in the form of the associated Legendre equation with the result that $k_{\mathrm{c}}$ and the corresponding eigenfunction $\phi_{\mathrm{c}}$ can be found easily (Laedke and Spatschek 1982; Munro and Parkes 2000). However, for intermediate values of $a$ we have not been able to find a transformation that will enable us to obtain the solution to the eigenvalue problem in closed form. Instead, we now obtain an approximate form for $k_{\mathrm{c}}(a)$ by making a perturbative expansion about the known values of $k_{\mathrm{c}}$ and the corresponding eigenfunctions at $a=0$ and $a=1$. The results are combined via a Padé approximant to give an approximate expression for $k_{\mathrm{c}}$ for all $0 \leqslant a \leqslant 1$.

For the small- $a$ expansion, we write (4.1) in the form

$$
\left[\frac{d^{2}}{d x^{2}}+\frac{\left(12+3 a^{2}\right) S^{2}+15 a S}{(1+a S)^{2}}-4-k_{\mathrm{c}}^{2}\right] \phi_{\mathrm{c}}=0
$$

in which $S \equiv \operatorname{sech} x$. For the ZK equation $k_{\mathrm{c}}=\sqrt{5}$ and $\phi_{\mathrm{c}}=\operatorname{sech}^{3} x$. We therefore substitute

$$
k_{\mathrm{c}}^{2}=5-\kappa_{1} a-\kappa_{2} a^{2}-\cdots
$$

and

$$
\phi_{\mathrm{c}}=S^{3}+a f_{1}(x)+a^{2} f_{2}(x)+\cdots
$$


into (4.2). At lowest order the expansion gives the $a=0$ result $L_{3} S^{3}=0$ where

$$
L_{3} \equiv \frac{d^{2}}{d x^{2}}+12 \operatorname{sech}^{2} x-9 .
$$

At first order in $a$ we find

$$
L_{3} f_{1}=\left(24 S^{3}-15 S-\kappa_{1}\right) S^{3} .
$$

Multiplying the above equation by $S^{3}$, integrating over all $x$, and using the selfadjoint property of $L_{3}$, the left-hand side vanishes and we are left with

$$
\kappa_{1}=\frac{225 \pi}{128} .
$$

In Sec. 5 we will also require $f_{1}$. Applying the inverse of $L_{3}$ to (4.5) and using (4.6), with the help of Mathematica one obtains

$$
\begin{aligned}
f_{1}= & \frac{5}{8}\left(\Upsilon \sinh x-\frac{\pi}{4} \cosh x+\frac{1}{2}\right) \cosh ^{2} x+\frac{25}{32} \Upsilon \sinh x-\frac{15}{128} \pi \cosh x-\frac{55}{192} \\
& -\frac{45}{256} \pi S+\frac{53}{128} S^{2}-3 S^{4}+\frac{75}{64}\left(\left(x S^{3}+S \tanh x\right) \Upsilon-\frac{S^{3}}{2} \int_{0}^{x} x^{\prime} \operatorname{sech} x^{\prime} d x^{\prime}\right)
\end{aligned}
$$

in which $\Upsilon \equiv \tan ^{-1} \tanh (x / 2)$. Note that although the first six terms in (4.7) do not vanish for large $|x|$ individually, it can be shown that the sum of those terms does tend to zero as $|x| \rightarrow \infty$ and hence so does $f_{1}(x)$.

To expand about the $a=1$ solution to the eigenvalue problem, we introduce $\bar{a}=1-a$. It is also more convenient to change the independent variable to $\bar{x}=x / 2$ after which (4.1) becomes

$$
\left[\frac{d^{2}}{d \bar{x}^{2}}+\frac{30(1-\bar{a}) \bar{S}^{2}+3 \bar{a}(3+\bar{a}) \bar{S}^{4}}{\left(1-\frac{1}{2} \bar{a} \bar{S}^{2}\right)^{2}}-16-4 k_{\mathrm{c}}^{2}\right] \phi_{\mathrm{c}}=0 .
$$

From the rescaled results of Munro and Parkes (2000) for the SZK equation, at $\bar{a}=0$ we have $k_{\mathrm{c}}^{2}=9 / 4$ and $\phi_{\mathrm{c}}=\operatorname{sech}^{5} \bar{x}$. We therefore make the expansions

$$
\begin{gathered}
k_{\mathrm{c}}^{2}=\frac{9}{4}+\bar{\kappa}_{1} \bar{a}+\bar{\kappa}_{2} \bar{a}^{2}+\cdots \\
\phi_{\mathrm{c}}=\bar{S}^{5}+\bar{a} \bar{f}_{1}(\bar{x})+\bar{a}^{2} \bar{f}_{2}(\bar{x})+\cdots
\end{gathered}
$$

and after substitution into (4.8) obtain $\bar{L}_{5} \bar{S}^{5}=0$, where

$$
\bar{L}_{5} \equiv \frac{d^{2}}{d \bar{x}^{2}}+30 \bar{S}^{2}-25
$$

at zeroth order in $\bar{a}$, and

$$
\bar{L}_{5} \bar{f}_{1}=\left(30 \bar{S}^{2}-39 \bar{S}^{4}+4 \bar{\kappa}_{1}\right) \bar{S}^{5}
$$

at first order. Multiplying this equation by $\bar{S}^{5}$ and integrating over all $\bar{x}$ so that the left-hand side vanishes yields

$$
\bar{\kappa}_{1}=\frac{15}{11}
$$


Again, we will require $\bar{f}_{1}$ for finding the growth rate for $k$ near $k_{\mathrm{c}}$. This time, applying $\bar{L}_{5}^{-1}$ to the first-order equation gives the comparatively simple expression,

$$
\bar{f}_{1}=\frac{3}{2} \bar{S}^{7}-\frac{6}{11} \bar{S}^{5} \ln \cosh \bar{x} .
$$

To obtain an expression for $k_{\mathrm{c}}$ for all $a$, we combine the results via a Padé approximant of the form

$$
k_{\mathrm{c}}^{2}(a)=a k_{\mathrm{c}}^{2}(1)+(1-a) \frac{k_{\mathrm{c}}^{2}(0)+c_{1} a}{1+d_{1} a} .
$$

Matching the small- $a$ and small- $\bar{a}$ expansions of (4.13) up to first order with (4.3) and (4.9), respectively, we obtain

$$
-\kappa_{1}=c_{1}+k_{\mathrm{c}}^{2}(1)-\left(1+d_{1}\right) k_{\mathrm{c}}^{2}(0), \quad \bar{\kappa}_{1}=\frac{k_{\mathrm{c}}^{2}(0)+c_{1}}{1+d_{1}}-k_{\mathrm{c}}^{2}(1) .
$$

Using the values of $k_{\mathrm{e}}$ at $a=0$ and $a=1$ and results (4.6) and (4.11) gives

$$
c_{1}=\frac{35775 \pi-95008}{7808}=2.22624, \quad d_{1}=\frac{2475 \pi}{1952}-\frac{182}{61}=0.999714
$$

It is very satisfying that this approximation method generates a value of $d_{1}$ so close to unity - it can be seen that singular behaviour in (4.13) occurs for $a=-1 / d_{1}$ which evidently corresponds to the fact that the conventional solitary wave solution (3.2) becomes singular as $a \rightarrow-1$.

By continuing the small- $a$ and small- $\bar{a}$ expansions it would be possible to obtain $\kappa_{2}$ and $\bar{\kappa}_{2}$ which would then allow us to calculate the coefficients in a higher order Padé approximant. However, as will be seen in Sec. 6, expression (4.13) is already an excellent approximation.

\section{Growth rate for $k \simeq k_{\mathrm{c}}$ instabilities}

To find the growth rate for $k$ near $k_{\mathrm{c}}$, we introduce the small parameter $K \equiv k_{\mathrm{c}}-k$, and then carry out small- $K$ expansions about the small- $a$ and small- $\bar{a}$ expansions of $\phi_{\mathrm{c}}$ we obtained earlier. Rewriting (3.4) in terms of $K$, we have

$$
\frac{d}{d x}\left(L-k_{\mathrm{e}}^{2}\right) \phi=-\gamma \phi+\left(K^{2}-2 k_{\mathrm{c}} K\right) \phi_{x}
$$

in which we expand $\phi$ and $\gamma$ as

$$
\phi=\phi_{\mathrm{c}}+K \Phi_{1}+K^{2} \Phi_{2}+\cdots
$$

and

$$
\gamma=\Gamma_{1} K+\Gamma_{2} K^{2}+\cdots
$$

Since we do not have $k_{\mathrm{c}}$ and $\phi_{\mathrm{c}}$ in closed form, we cannot find an exact expression for $\Gamma_{1}$ for arbitrary $a$ as was done for $\gamma_{1}$. Instead, by carrying out the expansion about the small- $a$ and small- $\bar{a}$ expressions for $\phi_{\mathrm{c}}$, we will obtain $\Gamma_{1}$ in the forms

$$
\Gamma_{1}(a)=\Gamma_{1}(0)\left(1-\mu_{1} a\right)+O\left(a^{2}\right), \quad \Gamma_{1}(a)=\Gamma_{1}(1)\left(1+\bar{\mu}_{1} \bar{a}\right)+O\left(\bar{a}^{2}\right),
$$

respectively. These will then be combined using a Padé approximant.

To zeroth order in $K,(5.1)$ reduces to (4.1). Equating coefficients of order $K$ in (5.1), integrating from $\infty$ to $x$, and using the result that $\phi_{\mathrm{c}}$ is even and vanishes at 
infinity, we have

$$
\left(L-k_{\mathrm{c}}^{2}\right) \Phi_{1}=\Gamma_{1}\left(\frac{1}{2}\left\langle\phi_{\mathrm{c}}\right\rangle-\int_{0}^{x} \phi_{\mathrm{c}}\left(x^{\prime}\right) d x^{\prime}\right)-2 k_{\mathrm{c}} \phi_{\mathrm{c}}
$$

where $\langle\cdot\rangle$ denotes the integral over all $x$. Multiplying (5.4) by $\phi_{\mathrm{c}}$ and integrating over all $x$, the left-hand side and the second term on the right-hand side vanish and we are left with

$$
\Gamma_{1}=\frac{4 k_{\mathrm{c}}\left\langle\phi_{\mathrm{c}}^{2}\right\rangle}{\left\langle\phi_{\mathrm{c}}\right\rangle^{2}}
$$

Note that this method of obtaining $\Gamma_{1}$ is more direct than that used previously (AR93; Munro and Parkes 2000).

Substituting the expansions (4.3) and (4.4) into (5.5) gives, to first order in $a$,

$$
\Gamma_{1}=\frac{4 \sqrt{5}\left\langle S^{6}\right\rangle}{\left\langle S^{3}\right\rangle^{2}}\left\{1-2\left(\frac{\kappa_{1}}{20}+\frac{\left\langle f_{1}\right\rangle}{\left\langle S^{3}\right\rangle}-\frac{\left\langle S^{3} f_{1}\right\rangle}{\left\langle S^{6}\right\rangle}\right) a\right\}+O\left(a^{2}\right) .
$$

Although the integral in the final term in (4.7) can be expressed in terms of polylogarithms, we have not been able to find the integral of this term over all $x$ analytically. Hence, the contributions of this last term to both $\left\langle f_{1}\right\rangle$ and $\left\langle S^{3} f_{1}\right\rangle$ were obtained numerically. Evaluating the integrals gives

$$
\Gamma_{1}(0)=\frac{256 \sqrt{5}}{15 \pi^{2}}, \quad \mu_{1}=1.09834
$$

Expression (5.5) is valid for all $a$ and so we may also use it to calculate the small- $K$ growth rate for small $\bar{a}$. Using (4.9) and (4.10) we obtain

$$
\Gamma_{1}=\frac{6\left\langle\bar{S}^{10}\right\rangle}{\left\langle\bar{S}^{5}\right\rangle^{2}}\left\{1+2\left(\frac{\bar{\kappa}_{1}}{9}-\frac{\left\langle\bar{f}_{1}\right\rangle}{\left\langle\bar{S}^{5}\right\rangle}+\frac{\left\langle\bar{S}^{5} \bar{f}_{1}\right\rangle}{\left\langle\bar{S}^{10}\right\rangle}\right) \bar{a}\right\}+O\left(\bar{a}^{2}\right)
$$

in which the integrals are still over $x$, not $\bar{x}$. In this case there are no difficulties in performing the integrals analytically and from the above expression we obtain

$$
\Gamma_{1}(1)=\frac{16384}{945 \pi^{2}}, \quad \bar{\mu}_{1}=\frac{6}{385}(140 \ln 2-59) .
$$

Both $\Gamma_{1}(0)$ and $\Gamma_{1}(1)$ agree with the values of $\bar{\gamma}_{2}$ obtained in AR93 and Munro and Parkes (2000), respectively, after allowing for the different expansions and normalizations in their calculations.

Finally we combine the small- $a$ and small- $\bar{a}$ results via a Padé approximant of the form

$$
\Gamma_{1 \mathrm{P}}=a \Gamma_{1}(1)+(1-a)\left(\frac{\Gamma_{1}(0)+g_{1} a}{1+h_{1} a}\right)
$$

which is of the same form as (4.13) for $k_{\mathrm{c}}^{2}$. As a result, the expressions for the Padé coefficients $g_{1}$ and $h_{1}$ are determined in an analogous way, and using (5.6) and (5.7) we find $g_{1}=1.72898$ and $h_{1}=0.999805$. Again, the fact that $h_{1}$ is so close to 1 shows that the approximant is reflecting the singular nature of the solitary wave solution for $a \rightarrow-1$.

\section{Numerical results and Padé approximant}

We have calculated the growth rate numerically using the shooting method technique given in AR93. Using the Newton-Raphson technique to obtain the value of 

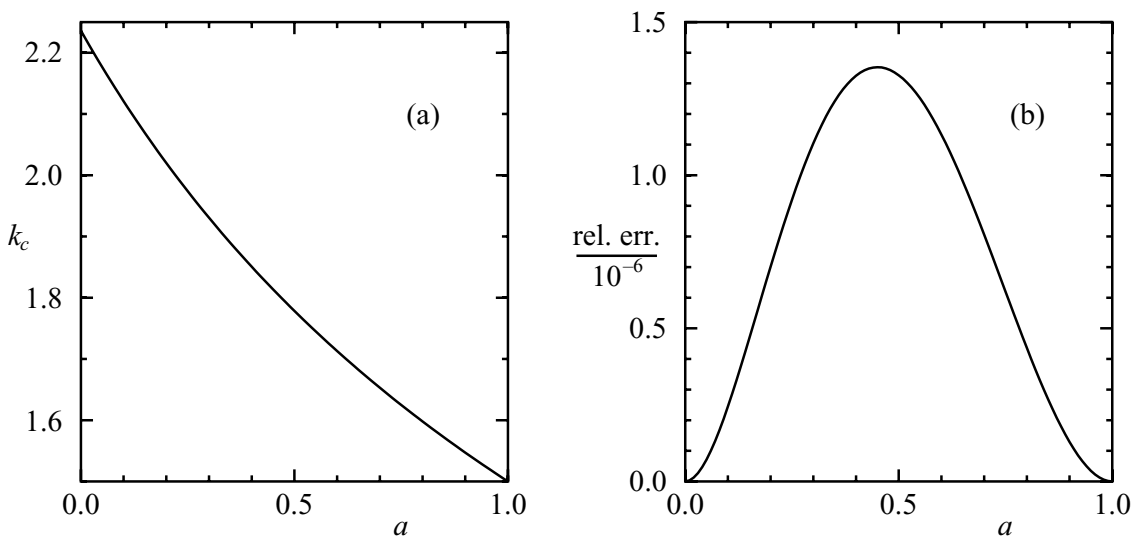

Figure 3. The dependence of $k_{\mathrm{c}}$ on $a$ : (a) numerically obtained $k_{\mathrm{c}}$; (b) relative error in the Padé approximant.

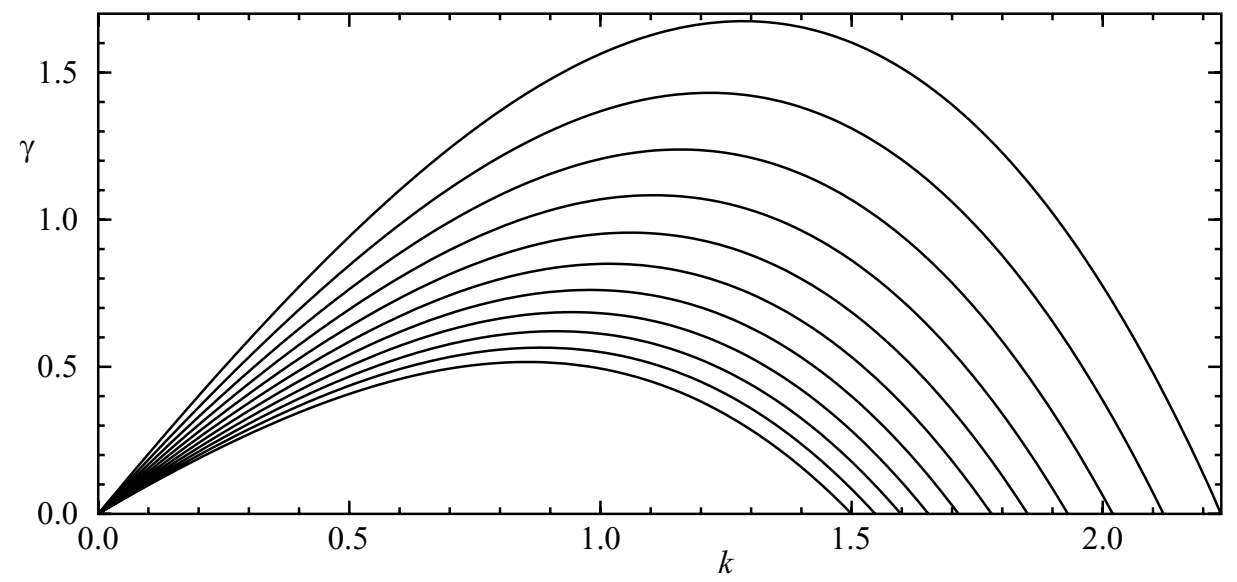

Figure 4. Growth rate curves for $a=0$ (top curve) to $a=1$ (lowest curve) in steps of 0.1 .

$k$ at which the numerically derived growth rate is zero, we obtain $k_{\mathrm{c}}$ as a function of $a$ as shown in Fig. 3(a). The Padé approximant for $k_{\mathrm{c}},(4.13)$, is very close to the numerical values of $k_{\mathrm{c}}$ for $0 \leqslant a \leqslant 1$, as is illustrated in Fig. 3(b). The maximum relative error between the two is $1.4 \times 10^{-6}$. The growth rate curves for various values of $a$ are shown in Fig. 4. It can be seen that as the electron distribution becomes less isothermal (as $a$ increases), not only is there a narrowing in the range of wavenumbers for which solitary waves are unstable, but also the growth rate of the instabilities decreases.

We can obtain an approximate analytical expression for the growth rate for all $k$ and $a$ within the region of instability in terms of a Padé approximant. As in AR93 we use a two-point approximant of the form

$$
\gamma_{\mathrm{P}}=\gamma_{1} k\left(1-k^{2} / k_{\mathrm{c}}^{2}\right)\left(\frac{1+p_{1} k}{1+q_{1} k}\right) .
$$

However, in this case $\gamma_{1}, k_{\mathrm{c}}, p_{1}$ and $q_{1}$ are functions of $a$. The form of the approximant has been chosen to vanish at $k=0$ and $k=k_{\mathrm{c}}$ and to have the correct 


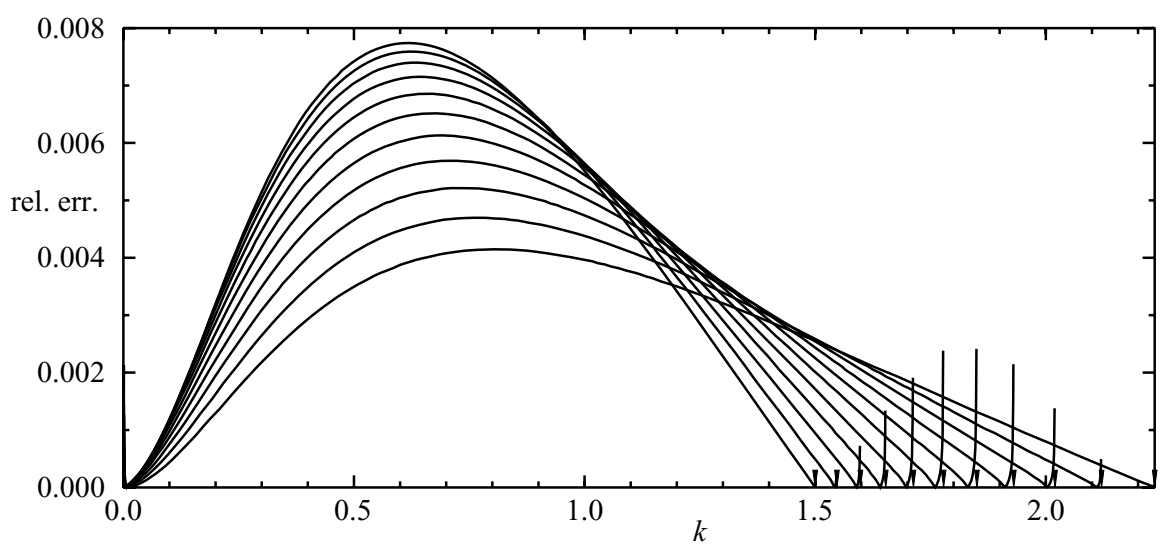

Figure 5. Relative error of Padé approximant $\gamma_{\mathrm{P}}$ compared with $\gamma_{\mathrm{N}}$ for $a=1$ (top curve) to $a=0$ (lowest curve) in steps of 0.1 . The wedges mark the values of $k_{\mathrm{c}}$ for each $a$.

first-order behaviour for small $k$. Matching its expansion at second order in $k$ and first order in $K$ with (3.5) and (5.2), respectively, yields

$$
p_{1}=\frac{\Gamma_{1} \gamma_{2}}{\gamma_{1}\left(\Gamma_{1}-2 \gamma_{1}\right)}-\frac{1}{k_{\mathrm{c}}}, \quad q_{1}=\frac{2 \gamma_{2}}{\Gamma_{1}-2 \gamma_{1}}-\frac{1}{k_{\mathrm{c}}} .
$$

Both $k_{\mathrm{c}}$ and $\Gamma_{1}$ are given by the Padé approximants we have already obtained. As we have not been able to calculate $\gamma_{2}$, we approximate it by linearly interpolating between its known values of $-4 / 15$ at $a=0$ (from AR93) and $-20 / 63$ at $a=1$ (obtained from Munro and Parkes (2000) after appropriate rescaling). This procedure is justified by the fact that, as can be seen in Fig. 5, the largest relative error in $\gamma_{\mathrm{P}}$ compared to the numerical value, $\gamma_{\mathrm{N}}$, occurs when $a=1$ at which point there is no interpolation error from $\gamma_{2}$. On the other hand, replacing (5.8) by a linearly interpolated form for $\Gamma_{1}$ in (6.2) leads to a $\gamma_{\mathrm{P}}$ with a much more significant deviation from $\gamma_{N}$. The relative error (given by $\left|\gamma_{P}-\gamma_{N}\right| / \gamma_{N}$ ) does not exceed $0.8 \%$ except for values of $k$ very close to $k_{\mathrm{c}}$ when $a$ is not 0 or 1 . These spikes in the error result from the fact that our expression for $k_{\mathrm{c}}$ is not exact.

\section{Conclusions}

In this article we have considered ion-acoustic solitary waves that propagate in the direction of the magnetic field in a strongly magnetized plasma which has a nonisothermal electron distribution. The system is governed by the SKdVZK equation, a modified form of the Zakharov-Kuznetsov equation with two nonlinear terms. We have obtained new algebraic and rarefactive solitary wave solutions to this equation, but chose to mostly study the stability with respect to perpendicular perturbations of the solitary wave solution that reduces to the $\mathrm{KdV}$ soliton in the isothermal limit. As the equation contains two nonlinear terms, the calculation of the growth rate is more involved than previous work concerning equations with a single nonlinearity. In particular, there is no exact analytical expression for the cutoff wavenumber, $k_{\mathrm{c}}$, or the corresponding eigenfunction. A method for obtaining approximate analytic forms for both quantities has been discussed and stability analysis for $k \simeq k_{\mathrm{c}}$ has been developed. These results have been combined with 
those of a small- $k$ stability analysis to obtain the variation of the growth rate with $k$ for $0 \leqslant k \leqslant k_{\mathrm{c}}$. They agree with the numerical results to within $0.8 \%$ except for $k$ extremely close to $k_{\mathrm{e}}$ where the growth rate is, in any case, extremely small.

The solitary wave solutions examined have been shown to be unstable to perpendicular perturbations for wavenumbers in the range $0<k<k_{\mathrm{c}}$. It is found that as the assumed electron distribution becomes increasingly less peaked than the Maxwellian, the growth rate and the range of unstable wavenumbers both decrease. Our attention has been drawn by the referee to Schamel (1982), which treats the much more complex problem of the transverse stability of more general BGK modes. A comprehensive comparison is out of the scope of this article, but it is interesting that the growth rate curve given is qualitatively similar to ours.

The methods developed in this paper are applicable to any higher-dimensional generalizations of the $\mathrm{KdV}$ equation containing two nonlinear terms if the cutoff wavenumbers and corresponding eigenfunctions are known for the equations containing only a single nonlinearity. In addition to the solitary wave solutions discussed in this paper, the SKdVZK equation also possesses nonlinear periodic solutions. These solutions and their stability have been studied and the results will appear in Part 2.

\section{Acknowledgements}

Two of the authors (M.A.A. and S.P.) wish to acknowledge support from the Thai Research Fund (BRG/18/2543). G.R. thanks Mahidol University for their hospitality during his visit.

\section{Appendix A. Stability of algebraic solitary waves}

It was pointed out in the main part of this paper that if $b<0$, in the limit $\eta \rightarrow 0$ the solitary wave solution $(2.2)$ reduces to the algebraic solitary wave $(2.3)$. The stability analysis as applied directly to the algebraic solitary wave is simpler than for the conventional solitary wave and serves as a check. It proceeds in an entirely analogous way to the small- $k$ expansion given in Sec. 3. Since the algebraic solitary wave solution is stationary, we insert the ansatz (3.3) into the untransformed equation (1.3), this time with $u_{0}(x)$ given by (2.3). The linearized equation is still (3.4) but with

$$
Q \equiv \frac{48 B}{\left(B+x^{2}\right)^{2}}-\frac{30}{B+x^{2}}
$$

where $B \equiv 75 /\left(4 b^{2}\right)$. After obtaining the zeroth-order solution,

$$
\phi_{0}=\frac{x}{\left(B+x^{2}\right)^{3}}
$$

and then $\phi_{1}$, the consistency condition at second order yields

$$
\gamma_{1}^{2}=\frac{56}{3 B^{2}} \sin ^{2} \theta
$$

This shows that the algebraic solitary wave is marginally stable for $\theta=0$ which is compatible with the results of our numerical experiments. Note that as a check on our calculation, we obtain exactly this expression on taking the limit as $\eta \rightarrow 0$ for $b<0$ of $\gamma_{1}^{2}$ as given by (3.7). 


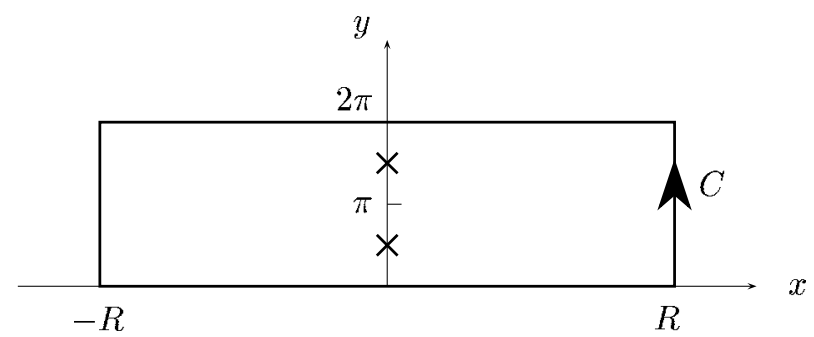

Figure B.1. Contour used in the evaluation of integrals of the form (B 1). The crosses mark the simple poles inside the contour.

Using the results of Sec. 4 we can obtain an estimate of the cut-off wavenumber for algebraic solitary wave instabilities. The parameter $\eta$, which was scaled away to simplify the analysis, can be reinstated by multiplying the expression for $k_{\mathrm{c}}^{2}$ in (4.13) by $\eta^{2}$. Then taking the limit as $\eta \rightarrow 0$ for $b<0$ gives

$$
k_{\mathrm{c}}^{2}=\frac{16\left(5-c_{1}\right) b^{2}}{75} \text {. }
$$

\section{Appendix B. Evaluation of integrals}

To find integrals of the form

$$
I_{p}=\int_{-\infty}^{\infty} x^{p} F(x) d x, \quad p=0,1,2, \ldots,
$$

where

$$
F(x)=\frac{\cosh ^{m} x \sinh ^{n} x}{(a+\cosh x)^{q}}, \quad m, n=0,1,2, \ldots, \quad q>m+n,
$$

and $p+n$ is even, we consider the integral of $z^{p+1} F(z)$, where $z=x+i y$, around the contour $C$ shown in Fig. B.1 (Allen 2007). For $|a|<1$, the integrand has simple poles at $z_{ \pm} \equiv\left(\pi \pm \cos ^{-1} a\right) i$. Hence, using the residue theorem and the fact that $F(z)$ has period $2 \pi i$ and $x^{p+1} F(x)$ is odd, we have, after taking the limit as $R \rightarrow \infty$ so that the contributions from the vertical parts of the contour vanish,

$$
2 \pi i\left\{\operatorname{Res}\left(z^{p+1} F(z), z_{+}\right)+\operatorname{Res}\left(z^{p+1} F(z), z_{-}\right)\right\}=-\int_{-\infty}^{\infty}(x+2 \pi i)^{p+1} F(x) d x .
$$

In the cases $p=0,1$, the right-hand side of $(\mathrm{B} 2)$ reduces to $-2(p+1) \pi i I_{p}$.

\section{References}

Allen, M. A. 1994 The Evolution of Plane Solitons PhD Thesis, University of Warwick.

Allen, M. A. 2007 Amer. Math. Monthly (in press).

Allen, M. A. and Rowlands, G. 1993 J. Plasma Phys. 50, 413.

Bernstein, I. B., Greene, J. M. and Kruskal, M. D. 1957 Phys. Rev. 108, 546.

Das, G. C., Sarma, J., Gao, Y.-T. and Uberoi, C. 2000 Phys. Plasmas 7, 2374.

Das, G. C. and Sen, K. M. 1991 Contrib. Plasma Phys. 31, 647.

El-Labany, S. K. and El-Taibany, W. F. 2004 J. Plasma Phys. 70, 69.

Ghosh, G. and Das, K. P. 1998 J. Plasma Phys. 59, 333. 
Infeld, E. and Rowlands, G. 2000 Nonlinear Waves, Solitons and Chaos, 2nd edn. Cambridge: Cambridge University Press.

Laedke, E. W. and Spatschek, K. H. 1982 J. Plasma Phys. 28, 469.

Munro, S. and Parkes, E. J. 1999 J. Plasma Phys. 62, 305.

Munro, S. and Parkes, E. J. 2000 J. Plasma Phys. 64, 411.

Ono, H. 1976 J. Phys. Soc. Japan 41, 1817.

Pelinovsky, D. E. and Grimshaw, R. H. J. 1997 Phys. Lett. A 229, 165.

Ramani, A. and Grammaticos, B. 1991 J. Phys. A: Math. Gen. 24, 1969.

Schamel, H. 1972 Plasma Phys. 14, 905.

Schamel, H. 1973 J. Plasma Phys. 9, 377.

Schamel, H. 1982 Phys. Rev. Lett. 48, 481.

Schamel, H. 1986 Phys. Rep. 140, 161.

Schamel, H. and Fedele, R. 2000 Phys. Plasmas 7, 3421.

Shukla, P. K. and Bharuthram, R. 1986 Phys. Rev. A 34, 4457.

Verheest, F., Mace, R. L., Pillay, S. R. and Hellberg, M. A. 2002 J. Phys. A: Math. Gen. 35, 795.

Washimi, H. and Taniuti, T. 1966 Phys. Rev. Lett. 17, 996.

Zakharov, V. E. and Kuznetsov, E. A. 1974 Sov. Phys. JETP 39, 285. 